\title{
Rogue Wave for the (3+1)-Dimensional Yu-Toda-Sasa-Fukuyama Equation
}

\author{
Hanlin Chen, ${ }^{1} \mathrm{Zhenhui}_{\mathrm{Xu}}{ }^{2}$ and Zhengde $\mathrm{Dai}^{3}$ \\ ${ }^{1}$ Joint Laboratory for Extreme Conditions Matter Properties, Southwest University of Science and Technology, Mianyang 621010, China \\ ${ }^{2}$ Applied Technology College, Southwest University of Science and Technology, Mianyang 621010, China \\ ${ }^{3}$ School of Mathematics and Physics, Yunnan University, Kunming 650091, China \\ Correspondence should be addressed to Zhenhui Xu; xuzhenhui19@163.com
}

Received 27 March 2014; Accepted 3 July 2014; Published 17 July 2014

Academic Editor: Qi-Ru Wang

Copyright (C) 2014 Hanlin Chen et al. This is an open access article distributed under the Creative Commons Attribution License, which permits unrestricted use, distribution, and reproduction in any medium, provided the original work is properly cited.

\begin{abstract}
A new method, homoclinic (heteroclinic) breather limit method (HBLM), for seeking rogue wave solution to nonlinear evolution equation (NEE) is proposed. (3+1)-dimensional Yu-Toda-Sasa-Fukuyama (YTSF) equation is used as an example to illustrate the effectiveness of the suggested method. A new family of two-wave solution, rational breather wave solution, is obtained by extended homoclinic test method, and it is just a rogue wave solution. This result shows rogue wave can come from extreme behavior of breather solitary wave for $(3+1)$-dimensional nonlinear wave fields.
\end{abstract}

\section{Introduction}

It is well known that solitary wave solutions of nonlinear evolution equations play an important role in nonlinear science fields, especially in nonlinear physical science, since they can provide much physical information and more insight into the physical aspects of the problem and thus lead to further applications [1]. In recent years, rogue waves, as a special type of nonlinear waves and also known as freak waves, monster waves, killer waves, extreme waves, and abnormal waves [2], have triggered much interest in various physical branches. Rogue wave is a kind of waves that seems abnormal which is first observed in the deep ocean. It always has two to three times amplitude higher than its surrounding waves and generally forms in a short time for which people think that it comes from nowhere. Rogue waves have been the subject of intensive research in oceanography $[3,4]$, optical fibres [57], superfluids [8], Bose-Einstein condensates, financial markets, and other related fields [9-13]. The first-order rational solution of the self-focusing nonlinear Schrödinger equation (NLS) was first found by Peregrine to describe the rogue waves phenomenon [14]. Recently, by using the Darboux dressing technique or Hirotas bilinear method, rogue waves solutions in complex system were obtained such as nonlinear
Schrödinger equation, Hirota equation, Sasa-Satsuma equation, Davey-Stewartson equation, coupled Gross-Pitaevskii equation, coupled NLS Maxwell-Bloch equation, and coupled Schrödinger-Boussinesq equation [15-26]. In this work, we propose a homoclinic (heteroclinic) breather limit method for seeking rogue wave solution to real NEE. We consider a general nonlinear partial differential equation in the form

$$
P\left(u, u_{t}, u_{x}, u_{y}, \ldots\right)=0,
$$

where $P$ is a polynomial in its arguments, $u: R_{x} \times R_{y} \times R_{t} \rightarrow$ $R$. To determine $u(t, x, y)$ explicitly, we take the following four steps.

Step 1. By Painlev'e analysis, a transformation

$$
u=T(f)
$$

is made for some new and unknown function $f$.

Step 2. By using the transformation in Step 1, original equation can be converted into Hirota's bilinear form

$$
G\left(D_{t}, D_{y} ; f\right)=0
$$


where the $D$-operator [27] is defined by

$$
\begin{aligned}
& D_{t}^{m} D_{y}^{n} f(t, y) \cdot g(t, y) \\
& \quad=\left.\left(\frac{\partial}{\partial t}-\frac{\partial}{\partial t^{\prime}}\right)^{m}\left(\frac{\partial}{\partial y}-\frac{\partial}{\partial y^{\prime}}\right)^{n}\left[f(t, y) g\left(t^{\prime}, y^{\prime}\right)\right]\right|_{t^{\prime}=t, y^{\prime}=y} .
\end{aligned}
$$

Step 3. Solve the above equation to get homoclinic (heteroclinic) breather wave solution by using extended homoclinic test approach (EHTA) [28].

Step 4. Letting the period of periodic wave go to infinite in homoclinic (heteroclinic) breather wave solution, we can obtain a rational homoclinic (heteroclinic) wave and this wave is just a rogue wave.

As a example we consider (3+1)-D Yu-Toda-SasaFukuyama equation which is an extension of BogoyavlenskiiSchiff (BS) equation in higher dimension [29]. It is well known that BS equation is the reduction of the self-dual YangMills equation; it is an integrable system and has an infinite number of conservation laws and $N$-soliton solutions [30]. tion is

The (3+1)-dimensional Yu-Toda-Sasa-Fukuyama equa-

$$
\begin{gathered}
\left(-4 u_{t}+\Phi(u) u_{z}\right)_{x}+3 u_{y y}=0 \\
\Phi(u)=\partial_{x}^{2}+4 u+2 u_{x} \partial_{x}^{-1} .
\end{gathered}
$$

It is called Yu-Toda-Sasa-Fukuyama (YTSF) equation. YTSF equation is not integrable system [29-31]; it is firstly presented by Yu et al. using the strong symmetry [30, 32]. The nontravelling wave solution was found using auto-Backlund transformation and the generalized projective Riccati equation method [32-34]. Moreover, some soliton-like solutions and periodic solutions for potential YTSF equation were obtained by Hiriota's bilinear method, the tanh-coth method, expfunction method, homoclinic test approach, and extended homoclinic test approach [33-37], respectively. Recently, some analytic solutions for the $(3+1)$-dimensional potential Yu-Toda-Sasa-Fukuyama equation [38] and the $(2+1)-$ dimensional Ablowitz-Kaup-Newell-Segur equation [39] are obtained by Darvishi using the modified extended homoclinic test approach, some exact solutions of the nonlinear ZK-MEW, and the potential YTSF equations by Zayed and Arnous using the modified simple equation method [40]. Besides these, further result on soliton and its feature for (5) were not studied up to now.

This work focuses on rational breather wave and then rogue wave solutions. Applying HBLM to $(3+1)$-D YTSF equation we firstly get breather solitary solution and then obtain rational breather solution by letting periodic wave go to infinite in breather solitary solution. Finally, we show that this rational breather wave is just a rogue wave. This is the new physical phenomenon found out up to now.

\section{Rational Homoclinic Wave (Rogue Wave)}

Let $\xi=x+c z$ in (5); for simplicity we take constant $c>0(c<$ 0 is similar), notice that $\partial_{x}=\partial_{\xi}, \partial_{z}=c \partial_{\xi}$ and so $c=\partial_{z} \partial_{x}^{-1}$, then (5) can be converted into the following form:

$$
-4 u_{\xi t}+c u_{\xi}^{4}+3 c\left(u^{2}\right)_{\xi \xi}+3 u_{y y}=0
$$

Setting $\eta=\xi-b t=x+c z-b t$ in (6) gives

$$
3 u_{y y}+4 b u_{\eta \eta}+3 c\left(u^{2}\right)_{\eta \eta}+c u_{\eta \eta}=0 .
$$

Setting $\zeta=i y$ in $(7)$ gives

$$
3 u_{\zeta \zeta}-4 b u_{\eta \eta}-3 c\left(u^{2}\right)_{\eta \eta}-c u_{\eta \eta}=0 .
$$

It is easy to see that (7) has an equilibrium solution $u_{0}$ which is an arbitrary constant.

We suppose that

$$
u=u_{0}+2(\ln f)_{\eta \eta}
$$

where $f(\eta, \zeta)$ is unknown real function. Substituting (9) into (8) we obtain the following bilinear form:

$$
\left(3 D_{\zeta}^{2}-\left(4 b+6 c u_{0}\right) D_{\eta}^{2}-c D_{\eta}^{4}-A\right) f \cdot f=0,
$$

where $A$ is an integration constant, $D_{\eta}^{4} f \cdot f=2\left(f f_{4 \eta}-4 f_{\eta} f_{3 \eta}+\right.$ $\left.3 f_{2 \eta}^{2}\right), D_{\eta}^{2} f \cdot f=2\left(f_{\eta \eta} f-f_{\eta}^{2}\right)$. With regard to (9), using the homoclinic test technique we can seek the solution in the form

$$
f=e^{-p_{1}(\eta-\alpha \zeta)}+\delta_{1} \cos (p(\eta+\beta \zeta))+\delta_{2} e^{p_{1}(\eta-\alpha \zeta)},
$$

where $p_{1}, p, \delta_{1}, \delta_{2}$ are real constants to be determined and $\alpha, \beta$ are constants to be determined.

Substituting (10) into (9) we can get an algebraic equation of $e^{p_{1}(\eta-\alpha \zeta)}$. Then equating the coefficients of all powers of $e^{j p(\xi-\alpha \zeta)}(j=-1,0,1)$ to zero, we get

$$
\begin{aligned}
& 2 \delta_{1} c p p_{1}^{3}+\left(4 b+6 c u_{0}\right) \delta_{1} p p_{1}+3 \delta_{1} \alpha \beta p p_{1}-2 \delta_{1} c p^{3} p_{1} \\
& \quad=0 \\
& \left(4 b+6 c u_{0}\right) \delta_{1} \delta_{2} p p_{1}+2 \delta_{1} \delta_{2} p p_{1}^{3} \\
& \quad+3 \delta_{1} \delta_{2} \alpha \beta p p_{1}-2 \delta_{1} \delta_{2} c p^{3} p_{1}=0, \\
& 12 \delta_{2} \alpha^{2} p_{1}^{2}-4 \delta_{1}^{2} c p^{4}+\left(4 b+6 u_{0}\right) \delta_{1}^{2} p^{2}-3 \delta_{1}^{2} \beta^{2} p^{2} \\
& \quad-4\left(4 b+6 c u_{0}\right) \delta_{2} p_{1}^{2}-16 \delta_{2} c p_{1}^{4}=0, \\
& 6 \delta_{1} c p^{2} p_{1}^{2}-\left(4 b+6 u_{0}\right) \delta_{1} p_{1}^{2}+\left(4 b+6 u_{0}\right) \delta_{1} p^{2} \\
& \quad-\delta_{1} c p_{4}-\delta_{1} c p_{1}^{4}+3 \delta_{1} \alpha^{2} p_{1}^{2}-3 \delta_{1} \beta^{2} p^{2}=0, \\
& -\delta_{1} \delta_{2} c p_{1}^{4}+6 \delta_{1} \delta_{2} c p^{2} p_{1}^{2}-\left(4 b+6 c u_{0}\right) \delta_{1} \delta_{2} p_{1}^{2}-3 \delta_{1} \delta_{2} \beta^{2} p^{2} \\
& \quad-\delta_{1} \delta_{2} c p^{4}+\left(4 b+6 c u_{0}\right) \delta_{1} \delta_{2} p^{2}+3 \delta_{1} \delta_{2} \alpha^{2} p_{1}^{2}=0 .
\end{aligned}
$$


Take $p_{1}=p$; then (12) can be reduced into the following:

$$
\begin{gathered}
\left(4 b+6 c u_{0}\right) \delta_{1} p^{2}+3 \delta_{1} \alpha \beta p^{2}=0, \\
\left(4 b+6 c u_{0}\right) \delta_{1} \delta_{2} p^{2}+3 \delta_{1} \delta_{2} \alpha \beta p^{2}=0, \\
4 \delta_{1} c p^{4}+3 \delta_{1} \alpha^{2} p^{2}-3 \delta_{1} \beta^{2} p^{2}=0, \\
4 \delta_{1} \delta_{2} c p^{4}-3 \delta_{1} \delta_{2} \beta^{2} p^{2} \delta_{1}+3 \delta_{1} \delta_{2} \alpha^{2} p^{2}=0, \\
12 \delta_{2} \alpha^{2} p^{2}-4 \delta_{1}^{2} c p^{4}+\left(4 b+6 c u_{0}\right) \delta_{1}^{2} p^{2}-3 \delta_{1} \beta^{2} p^{2} \\
-4\left(4 b+6 c u_{0}\right) \delta_{2} p^{2}-16 \delta_{2} c p^{4}=0 .
\end{gathered}
$$

Solving (13) yields

$$
\begin{gathered}
\delta_{1}= \pm 2 \frac{\sqrt{\left(6 \alpha^{2}-2\left(2 b-3 u_{0}\right)-3 \beta^{2}\right) \delta_{2}}}{\sqrt{6 \beta^{2}-2\left(2 b-3 u_{0}\right)-3 \alpha^{2}}}, \\
\alpha \beta=-\frac{1}{3}\left(4 b+6 c u_{0}\right), \quad p^{2}=\frac{3}{4 c}\left(\beta^{2}-\alpha^{2}\right),
\end{gathered}
$$

where $b, c, \delta_{2}$ are some free real constants and $\alpha, \beta$ are some free constants. Setting $c=-1$ in (14) gives

$$
\begin{gathered}
\delta_{1}= \pm 2 \frac{\sqrt{\left(6 \alpha^{2}-2\left(2 b-3 u_{0}\right)-3 \beta^{2}\right) \delta_{2}}}{\sqrt{6 \beta^{2}-2\left(2 b-3 u_{0}\right)-3 \alpha^{2}}}, \\
\alpha \beta=-\frac{2}{3}\left(2 b-3 u_{0}\right), \quad p^{2}=\frac{3}{4}\left(\alpha^{2}-\beta^{2}\right) .
\end{gathered}
$$

Choosing $u_{0} \neq 2 b / 3$ and $\delta_{2}>0$, we get from (15)

$$
\begin{gathered}
|\beta|>|\alpha|, \quad \alpha=-\frac{2\left(2 b-3 u_{0}\right)}{3 \beta}, \\
\alpha^{2}>\frac{2\left(2 b-3 u_{0}\right)}{3 \beta}, \quad\left(u_{0}<\frac{2 b}{3} \text { or }>\frac{2 b}{3}\right) \\
\text { or } \alpha^{2}<\frac{2 b}{3} \cdot\left(u_{0}>\frac{2 b}{3}\right) .
\end{gathered}
$$

Substituting (15)-(16) into (11), we have

$$
\begin{aligned}
f_{1}(\eta, \zeta)= & 2 \sqrt{\delta_{2}} \cosh \left(p\left(\eta+\frac{2\left(2 b-3 u_{0}\right)}{3 \beta} \zeta\right)+\frac{1}{2} \ln \left(\delta_{2}\right)\right) \\
& +h_{1} \cos (p(\eta+\beta \zeta)), \\
f_{2}(\eta, \zeta)= & 2 \sqrt{\delta_{2}} \cosh \left(p\left(\eta+\frac{2\left(2 b-3 u_{0}\right)}{3 \beta} \zeta\right)+\frac{1}{2} \ln \left(\delta_{2}\right)\right) \\
& -h_{1} \cos (p(\eta+\beta \zeta)),
\end{aligned}
$$

where $h_{1}=2 \sqrt{\left(6 \alpha^{2}-2\left(2 b-3 u_{0}\right)-3 \beta^{2}\right) \delta_{2}}$ ， $\sqrt{6 \beta^{2}-2\left(2 b-3 u_{0}\right)-3 \alpha^{2}}, p= \pm\left(\sqrt{3\left(\alpha^{2}-\beta^{2}\right)} / 2\right)$, and $\alpha, \beta$ are some free constants. Substituting (17) into (9) yields the solutions of (8) as follows, respectivly:

$$
\begin{aligned}
& u_{1}(\eta, \zeta) \\
& =u_{0}+\left(2 p ^ { 2 } \left(m_{0}\right.\right.
\end{aligned}
$$$$
+2 m_{1} \sinh \left(p\left(\eta+\frac{2\left(2 b-3 u_{0}\right)}{3 \beta} \zeta\right)\right.
$$$$
\left.+\frac{1}{2} \ln \left(\delta_{2}\right)\right)
$$$$
\times \sin (p(\eta+\beta \zeta)))
$$

$$
\times\left(\left(\cosh \left(p\left(\eta+\frac{2\left(2 b-3 u_{0}\right)}{3 \beta} \zeta\right)+\frac{1}{2} \ln \left(\delta_{2}\right)\right)\right.\right.
$$

$$
\left.\left.\left.+m_{1} \cos (p(\eta+\beta \zeta))\right)^{2}\right)^{-1}\right)
$$

$u_{2}(\eta, \zeta)$

$=u_{0}+\left(2 p^{2}\left(m_{0}\right.\right.$

$$
\begin{gathered}
-2 m_{1} \sinh \left(p\left(\eta+\frac{2\left(2 b-3 u_{0}\right)}{3 \beta} \zeta\right)\right. \\
\left.+\frac{1}{2} \ln \left(\delta_{2}\right)\right) \\
\times \sin (p(\eta+\beta \zeta))) \\
\times\left(\left(\cosh \left(p\left(\eta+\frac{2\left(2 b-3 u_{0}\right)}{3 \beta} \zeta\right)+\frac{1}{2} \ln \left(\delta_{2}\right)\right)\right.\right. \\
\left.\left.\left.-m_{1} \cos (p(\eta+\beta \zeta))\right)^{2}\right)^{-1}\right),
\end{gathered}
$$

where $m_{0}=9\left(\beta^{2}-\alpha^{2}\right) /\left(6 \beta^{2}-2\left(2 b-3 u_{0}\right)-3 \alpha^{2}\right), m_{1}=$ $\sqrt{6 \alpha^{2}-2\left(2 b-3 u_{0}\right)-3 \beta^{2}} / \sqrt{6 \beta^{2}-2\left(2 b-3 u_{0}\right)-3 \alpha^{2}}<1$, and $p= \pm\left(\sqrt{3\left(\alpha^{2}-\beta^{2}\right)} / 2\right)$. 
Taking $\zeta=i y, \alpha=a i$, and $\beta=\omega i$ into (18) yields the solutions of (7) as follows, respectivly:

$$
\begin{aligned}
& u_{1}(\eta, y) \\
& =u_{0}+2 p^{2}\left(m_{0}\right. \\
& +2 m_{1} \sinh \left(p\left(\eta+\frac{2\left(2 b-3 u_{0}\right)}{3 \omega} y\right)\right. \\
& \quad \times \sin (p(\eta-\omega y))) \\
& \times\left(\left(\operatorname { c o s h } \left(p\left(\eta+\frac{1}{2} \ln \left(\delta_{2}\right)\right)\right.\right.\right. \\
& \left.\left.\left.\quad+m_{1} \cos (p(\eta-\omega y))\right)^{2}\right)^{-1}\right),
\end{aligned}
$$

$$
u_{2}(\eta, y)
$$$$
=u_{0}+\left(2 p ^ { 2 } \left(m_{0}\right.\right.
$$

$$
\begin{gathered}
-2 m_{1} \sinh \left(p\left(\eta+\frac{2\left(2 b-3 u_{0}\right)}{3 \omega} y\right)\right. \\
\left.+\frac{1}{2} \ln \left(\delta_{2}\right)\right) \\
\times \sin (p(\eta-\omega y))) \\
\times\left(\left(\cosh \left(p\left(\eta+\frac{2\left(2 b-3 u_{0}\right)}{3 \omega} y\right)+\frac{1}{2} \ln \left(\delta_{2}\right)\right)\right.\right. \\
\left.\left.\left.-m_{1} \cos (p(\eta-\omega y))\right)^{2}\right)^{-1}\right),
\end{gathered}
$$

where $a, \omega$ are some free real constants, $m_{0}=$ $9\left(\omega^{2}-a^{2}\right) /\left(6 \omega^{2}+2\left(2 b-3 u_{0}\right)-3 a^{2}\right), m_{1}=$ $\sqrt{6 a^{2}+2\left(2 b-3 u_{0}\right)-3 \omega^{2}} / \sqrt{6 \omega^{2}+2\left(2 b-3 u_{0}\right)-3 a^{2}}<1$, and $p= \pm\left(\sqrt{3\left(\omega^{2}-a^{2}\right)} / 2\right)$.

The solution $u_{1}(\eta, y)$ (resp., $u_{2}(\eta, y)$ ) shows a new family of two-wave, breather solitary wave, which is a solitary wave and meanwhile is a periodic wave whose amplitude periodically oscillates with the evolution of time. It shows elastic interaction between a left-propagation (backwarddirection) periodic wave with speed $b$ and homoclinic wave of different direction with speed $2\left(2 b-3 u_{0}\right) / 3 \omega$.
Taking $\eta=\xi-b t=x-z-b t$ into (19) gives and yields the breather-type soliton solutions of the (3+1)-D YTSF equation as follows, respectivly (see Figures 1 and 2):

$$
\begin{aligned}
& u_{1}(t, x, y, z) \\
& =u_{0}+\left(2 p ^ { 2 } \left(m_{0}\right.\right. \\
& +2 m_{1} \sinh \left(p\left(x+\frac{2\left(2 b-3 u_{0}\right)}{3 \omega} y-z-b t\right)\right. \\
& \left.+\frac{1}{2} \ln \left(\delta_{2}\right)\right) \\
& \times \sin (p(x-\omega y-z-b t))) \\
& \times\left(\left(\operatorname { c o s h } \left(p\left(x+\frac{2\left(2 b-3 u_{0}\right)}{3 \omega} y-z-b t\right)\right.\right.\right. \\
& \left.+\frac{1}{2} \ln \left(\delta_{2}\right)\right) \\
& \left.\left.\left.+m_{1} \cos (p(x-\omega y-z-b t))\right)^{2}\right)^{-1}\right), \\
& u_{2}(t, x, y, z) \\
& =u_{0}+\left(2 p ^ { 2 } \left(m_{0}\right.\right. \\
& -2 m_{1} \sinh \left(p\left(x+\frac{2\left(2 b-3 u_{0}\right)}{3 \omega} y-z-b t\right)\right. \\
& \left.+\frac{1}{2} \ln \left(\delta_{2}\right)\right) \\
& \times \sin (p(\eta-\omega y-z-b t))) \\
& \times\left(\left(\operatorname { c o s h } \left(p\left(x+\frac{2\left(2 b-3 u_{0}\right)}{3 \omega} y-z-b t\right)\right.\right.\right. \\
& \left.+\frac{1}{2} \ln \left(\delta_{2}\right)\right) \\
& \left.\left.\left.-m_{1} \cos (p(x-\omega y-z-b t))\right)^{2}\right)^{-1}\right)
\end{aligned}
$$

where $a, \omega$ are some free real constants, $m_{0}=$ $9\left(\omega^{2}-a^{2}\right) /\left(6 \omega^{2}+2\left(2 b-3 u_{0}\right)-3 a^{2}\right), m_{1}=$ $\sqrt{6 a^{2}+2\left(2 b-3 u_{0}\right)-3 \omega^{2}} / \sqrt{6 \omega^{2}+2\left(2 b-3 u_{0}\right)-3 a^{2}}<1$, and $p= \pm\left(\sqrt{3\left(\omega^{2}-a^{2}\right)} / 2\right)$. 


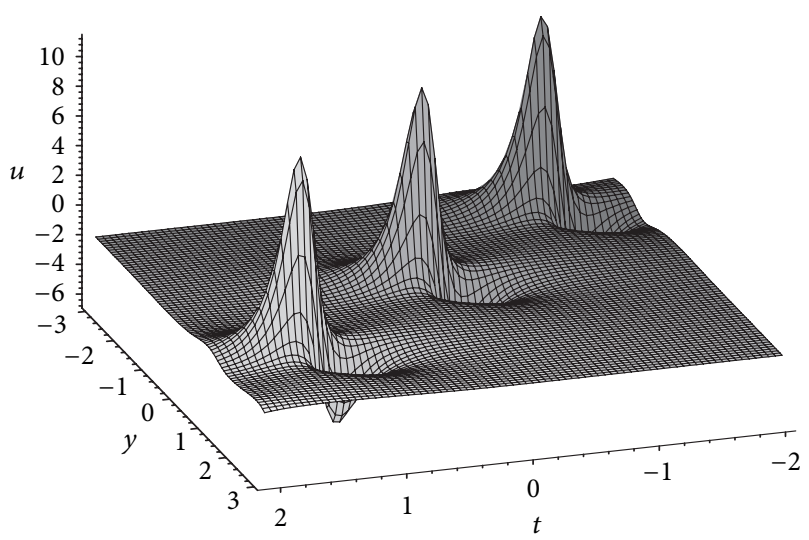

FIgURE 1: The figure of $u_{1}(t, x, y, z)$ as $u_{0}=-2$ and $b=3$.

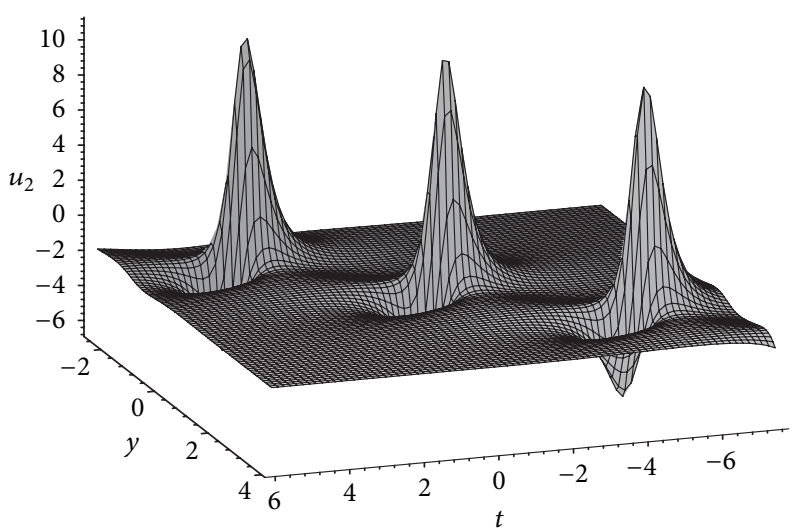

Figure 2: The figure of $u_{2}(t, x, y, z)$ as $u_{0}=-2, b=3, \omega=3.5$, $p=0.6092400758, x=-1$, and $z=-2$.

Use (19) and take $\delta_{2}=1$; then $(1 / 2) \ln \left(\delta_{2}\right)=0$ in $u_{2}$. So, solution $u_{2}$ can be rewritten as follows:

$$
\begin{aligned}
& u_{2}^{(1)}(\eta, y) \\
&=u_{0}+\left(2 p ^ { 2 } \left(m_{0}-2 m_{1} \sinh \left(p\left(\eta+\frac{2\left(2 b-3 u_{0}\right)}{3 \omega} y\right)\right)\right.\right. \\
&\quad \times \sin (p(\eta-\omega y))) \\
& \times\left(\left(\cosh \left(p\left(\eta+\frac{2\left(2 b-3 u_{0}\right)}{3 \omega} y\right)\right)\right.\right. \\
&\left.\left.\left.\quad-m_{1} \cos (p(\eta-\omega y))\right)^{2}\right)^{-1}\right),
\end{aligned}
$$

where $m_{0}=12 p^{2} /\left(4 p^{2}+2\left(2 b-3 u_{0}\right)+3 \omega^{2}\right)$ and $m_{1}=$ $\sqrt{3 a^{2}+2\left(2 b-3 u_{0}\right)-4 p^{2}} / \sqrt{4 p^{2}+2\left(2 b-3 u_{0}\right)+3 \omega^{2}}$.
Now we consider a limit behavior of $u_{2}^{(1)}$ as the period $2 \pi / p$ of periodic wave $\cos (p(\eta-\omega y))$ goes to infinite; that is, $p \rightarrow 0$. By computing, we obtain the following result:

$$
\begin{aligned}
& U_{\text {rogue wave }} \\
& =u_{0}+\frac{16\left(6 A-\left(\eta+\left(2\left(2 b-3 u_{0}\right) / 3 \omega\right) y\right)(\eta-\omega y)\right)}{\left(\left(\eta+\left(2\left(2 b-3 u_{0}\right) / 3 \omega\right) y\right)^{2}+(\eta-\omega y)^{2}+8 A\right)^{2}},
\end{aligned}
$$

where $A=1 /\left(3 \omega^{2}+2\left(2 b-3 u_{0}\right)\right)$; here we have used $m_{1} \rightarrow 1$ and $\omega=a$ as $p \rightarrow 0$.

$U$ contains two waves with different velocities and directions. It is easy to verify that $U_{\text {rogue wave }}$ is a rational solution of (7). Moreover, we can show that $U_{\text {rogue wave }}$ also is breathertype solution. In fact, $U \rightarrow 0$ for fixed $\eta$ as $y \rightarrow \pm \propto$. So, $U$ is not only a rational breather solution but also a rogue wave solution which has two to three times amplitude higher than its surrounding waves and generally forms in a short time. It is an example that the rogue wave can come from breather solitary wave solution for real equation. One can think whether the energy collection and superposition of breather solitary wave in many many periods leads to a rogue wave or not.

Taking $\eta=\xi-b t=x-z-b t$ into (21), we obtain the rogue wave solutions of the (3+1)-D YTSF equation as follows (see Figure 3):

$U_{\text {(ytsf) }}$ rogue wave

$$
\begin{gathered}
=u_{0}+\left(1 6 \left(6 A-\left(x+\frac{2\left(2 b-3 u_{0}\right)}{3 \omega} y-z-b t\right)\right.\right. \\
\times(x-\omega y-z-b t)) \\
\times\left(\left(\left(x+\frac{2\left(2 b-3 u_{0}\right)}{3 \omega} y-z-b t\right)^{2}\right.\right. \\
\left.\left.\left.+(x-\omega y-z-b t)^{2}+8 A\right)^{2}\right)^{-1}\right),
\end{gathered}
$$

where $A=1 /\left(3 \omega^{2}+2\left(2 b-3 u_{0}\right)\right)$; here we have used $m_{1} \rightarrow 1$ and $\omega=a$ as $p \rightarrow 0$.

\section{Conclusion}

In this paper, we propose a new method for seeking rogue wave, homoclinic (heteroclinic) breather limit method (HBLM). Applying this method to the real (3+1)-D YTSF equation, we obtain a family of homoclinic breather solution and rational homoclinic solution. Furthermore, rational homoclinic solution obtained here is just a rogue wave solution, and then we obtain the rogue wave solutions of the $(3+1)$-D YTSF equation. In future, we intend to study the interaction between breather wave and solitary wave. What 


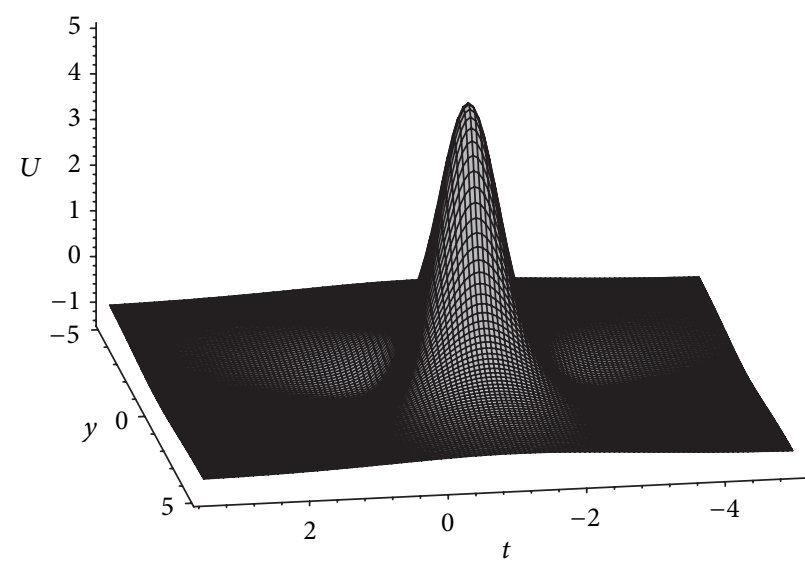

(a)

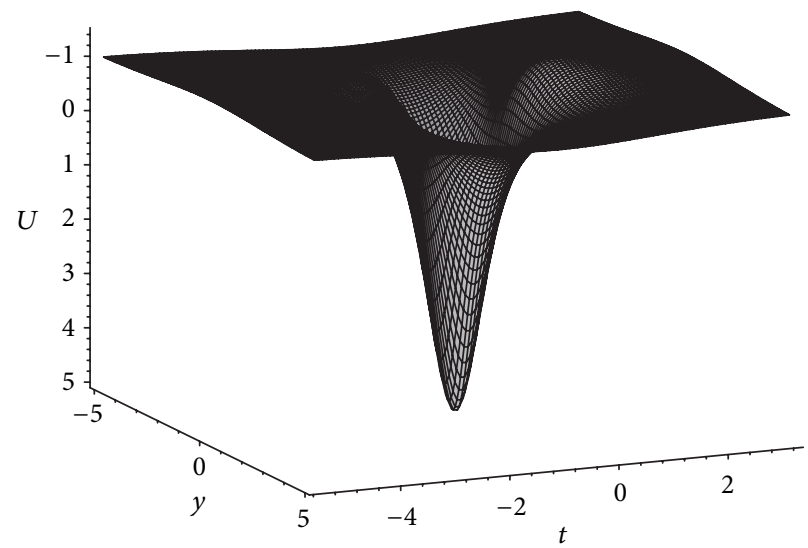

(b)

FIgURE 3: The figure of $U_{(\mathrm{ytsf})}$ rogue wave as $a=\omega, u_{0}=1, b=2, \omega=\sqrt{6} / 3, x=-1$, and $z=-2$.

is more, can we obtain similar results to another integrable or nonintegrable system with homoclinic or heteroclinic breather wave? How can one use the homoclinic breather wave to obtain rogue wave under contained conditions?

\section{Conflict of Interests}

The authors declare that there is no conflict of interests regarding the publication of this paper.

\section{Acknowledgments}

This work was supported by the Chinese Natural Science Foundation Grants nos. 11372294 and 11361048 and the Sichuan Educational science Foundation Grant no. 09zc008.

\section{References}

[1] M. J. Ablowitz and P. A. Clarkson, Solitons, Nonlinear Evolution Equations and Inverse Scattering, Cambridge University Press, 1991.

[2] Y. Ohta and J. Yang, "General high-order rogue waves and their dynamics in the nonlinear Schrödinger equation," Proceedings of The Royal Society of London A: Mathematical, Physical and Engineering Sciences, vol. 468, no. 2142, pp. 1716-1740, 2012.

[3] P. Müller, C. Garrett, and A. Osborne, "Rogue waves-the fourteenth 'Aha Huliko'a Hawaiian winter workshop," Oceanography, vol. 18, no. 3, pp. 66-75, 2005.

[4] C. Kharif, E. Pelinovsky, and A. Slunyaev, Rogue Waves in the Ocean, Observation, Theories and Modeling, Springer, New, 2009.

[5] N. Akhmediev, A. Ankiewicz, and J. M. Soto-Crespo, "Rogue waves and rational solutions of the nonlinear Schrödinger equation," Physical Review E: Statistical, Nonlinear, and Soft Matter Physics, vol. 80, no. 2, Article ID 026601, 2009.

[6] D. R. Solli, C. Ropers, P. Koonath, and B. Jalali, "Optical rogue waves," Nature, vol. 450, no. 7172, pp. 1054-1057, 2007.

[7] Y. V. Bludov, V. V. Konotop, and N. Akhmediev, "Rogue waves as spatial energy concentrators in arrays of nonlinear waveguides," Optics Letters, vol. 34, no. 19, pp. 3015-3017, 2009.
[8] A. N. Ganshin, V. B. Efimov, G. V. Kolmakov, L. P. MezhovDeglin, and P. V. E. McClintock, "Statistical properties of strongly nonlinear waves within a resonator," Physical Review Letters, vol. 101, Article ID 065303, 2008.

[9] Y. V. Bludov, V. V. Konotop, and N. Akhmediev, "Matter rogue waves," Physical Review A, vol. 80, no. 3, Article ID 033610, 2009.

[10] M. Onorato, S. Residori, U. Bortolozzo, A. Montina, and F. T. Arecchi, "Rogue waves and their generating mechanisms in different physical contexts," Physics Reports, vol. 528, no. 2, pp. 47-89, 2013.

[11] D. R. Solli, C. Ropers, and B. Jalali, "Active control of rogue waves for stimulated supercontinuum generation," Physical Review Letters, vol. 101, no. 23, Article ID 233902, 2008.

[12] N. Akhmediev, J. M. Soto-Crespo, and A. Ankiewicz, "Extreme waves that appear from nowhere: on the nature of rogue waves," Physics Letters A, vol. 373, no. 25, pp. 2137-2145, 2009.

[13] Z. Y. Yan, "Vector financial rogue waves," Physics Letters, Section A: General, Atomic and Solid State Physics, vol. 375, no. 48, pp. 4274-4279, 2011.

[14] D. H. Peregrine, "Water waves, nonlinear Schrödinger equations and their solutions," Australian Mathematical Society Journal B: Applied Mathematics, vol. 25, no. 1, pp. 16-43, 1983.

[15] Y. Tao and J. He, "Multisolitons, breathers, and rogue waves for the Hirota equation generated by the Darboux transformation," Physical Review E, vol. 85, no. 2, Article ID 026601, 2012.

[16] B. Guo, L. Ling, and Q. P. Liu, "Nonlinear schrödinger equation: generalized darboux transformation and rogue wave solutions," Physical Review E, vol. 85, no. 2, Article ID 026607, 2012.

[17] M. J. Ablowitz and J. Villarroel, "Solutions to the time dependent Schrödinger and the Kadomtsev-Petviashvili equations," Physical Review Letters, vol. 78, no. 4, pp. 570-573, 1997.

[18] U. Bandelow and N. Akhmediev, "Persistence of rogue waves in extended nonlinear Schrödinger equations: integrable SasaSatsuma case," Physics Letters, Section A: General, Atomic and Solid State Physics, vol. 376, no. 18, pp. 1558-1561, 2012.

[19] Y. Ohta and J. Yang, "Rogue waves in the Davey-Stewartson I equation,” Physical Review E, vol. 86, no. 3, Article ID 036604, 2012.

[20] Y. Ohta and J. Yang, "Dynamics of rogue waves in the DaveyStewartson II equation," Journal of Physics A: Mathematical and Theoretical, vol. 46, no. 10, Article ID 105202, 2013. 
[21] L. C. Zhao and J. Liu, "Rogue-wave solutions of a threecomponent coupled nonlinear Schrödinger equation," Physical Review E, vol. 87, no. 1, Article ID 013201, 2013.

[22] W. P. Zhong, "Rogue wave solutions of the generalized onedimensional Gross-Pitaevskii equation," Journal of Nonlinear Optical Physics and Materials, vol. 21, no. 2, Article ID 1250026, 2012.

[23] C. Li, J. He, and K. Porseizan, "Rogue waves of the Hirota and the Maxwell-Bloch equations," Physical Review E, vol. 87, no. 1, Article ID 012913, 2013.

[24] P. Gaillard, "Families of quasi-rational solutions of the NLS equation and multi-rogue waves," Journal of Physics A: Mathematical and Theoretical, vol. 44, no. 43, Article ID 435204, 2011.

[25] P. Dubard, P. Gaillard, C. Klein, and V. B. Matveev, "On multirogue wave solutions of the NLS equation and positon solutions of the KdV equation," The European Physical Journal: Special Topics, vol. 185, no. 1, pp. 247-258, 2010.

[26] G. Mu and Z. Qin, "Rogue waves for the coupled SchrödingerBoussinesq equation and the coupled Higgs equation," Journal of the Physical Society of Japan, vol. 81, no. 8, p. 4001, 2012.

[27] R. Hirota, "Fundamental properties of the binary operators in soliton theory and their generalization," in Dynamical Problem in Soliton Systems, S. Takeno, Ed., vol. 30 of Springer Series in Synergetiecs, Springer, Berlin, Germany, 1985.

[28] Z. Dai, J. Liu, and D. Li, "Applications of HTA and EHTA to YTSF equation," Applied Mathematics and Computation, vol. 207, no. 2, pp. 360-364, 2009.

[29] S. Yu, K. Toda, N. Sasa, and T. Fukuyama, "N soliton solutions to the Bogoyavlenskii-Schiff equation and a quest for the soliton solution in $(3+1)$ dimensions," Journal of Physics A. Mathematical and General, vol. 31, no. 14, pp. 3337-3347, 1998.

[30] J. Schff, Painleve Transendent, Their Asymptotics and Physical Applications, Plenum, New York, NY, USA, 1992.

[31] K. Toda, J. Y. Song, and T. Fukuyama, "The BogoyavlenskiiSchiff hierarchy and integrable equation in $(2+1)$-dimensions," Reports on Mathematical Physics, vol. 44, pp. 247-254, 1999.

[32] Z. Yan, "New families of nontravelling wave solutions to a new $(3+1)$-dimensional potential-YTSF equation," Physics Letters A, vol. 318, no. 1-2, pp. 78-83, 2003.

[33] X. Zeng, Z. Dai, and D. Li, "New periodic soliton solutions for the $(3+1)$-dimensional potential-YTSF equation," Chaos, Solitons \& Fractals, vol. 42, no. 2, pp. 657-661, 2009.

[34] T. Zhang, H. N. Xuan, D. Zhang, and C.-J. Wang, "Nontravelling wave solutions to a $(3+1)$-dimensional potentialYTSF equation and a simplified model for reacting mixtures," Chaos, Solitons and Fractals, vol. 34, no. 3, pp. 1006-1013, 2007.

[35] A. Wazwaz, "Multiple-soliton solutions for the CalogeroBogoyavlenskii-Schiff, Jimbo-Miwa and YTSF equations," Applied Mathematics and Computation, vol. 203, no. 2, pp. 592-597, 2008.

[36] A. Borhanifar and M. M. Kabir, "New periodic and soliton solutions by application of Exp-function method for nonlinear evolution equations," Journal of Computational and Applied Mathematics, vol. 229, no. 1, pp. 158-167, 2009.

[37] Z. Guo and J. Yu, "Multiplicity results on period solutions to higher dimensional differential equations with multiple delays," Journal of Dynamics and Differential Equations, vol. 23, no. 4, pp. 1029-1052, 2011.

[38] M. T. Darvishi and M. Najafi, "A modification of EHTA to solve the $(3+1)$ dimensional potential-YTFS equation," Chinese Physics Letters, vol. 28, Article ID 040202, 2011.
[39] M. Najafi and M. T. Darvishi, "New exact solutions to the $(2+1)$ dimensional Ablowitz-Kaup-Newell-Segur equation: Modification of the extended homoclinic test approach," Chinese Physics Letters, vol. 29, no. 4, Article ID 040202, 2012.

[40] E. M. E. Zayed and A. H. Arnous, "Exact solutions of the nonlinear ZK-MEW and the potential YTSF equations using the modified simple equation method," in Proceedings of the International Conference of Numerical Analysis and Applied Mathematics (ICNAAM '12), pp. 2044-2048, September 2012. 


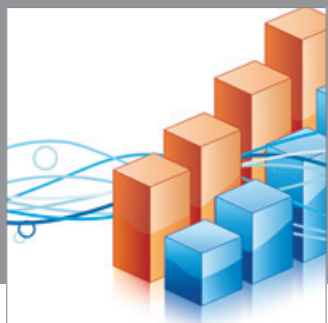

Advances in

Operations Research

mansans

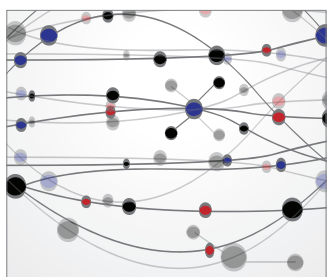

The Scientific World Journal
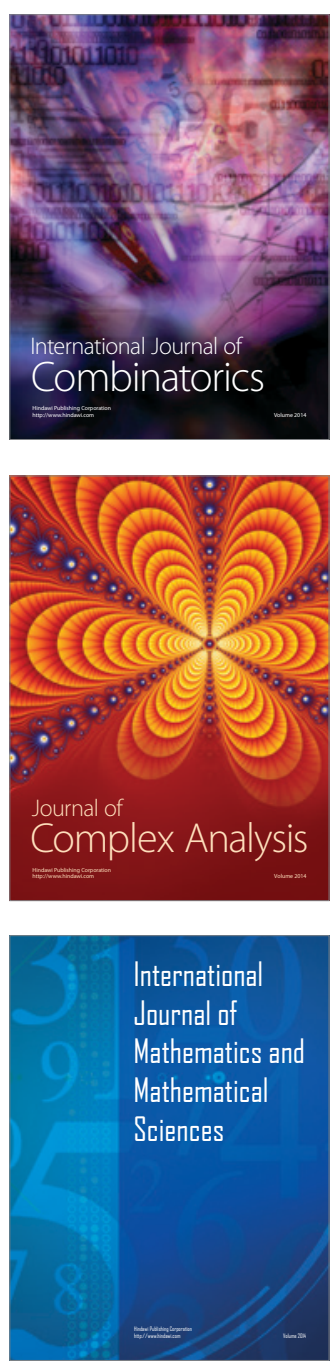
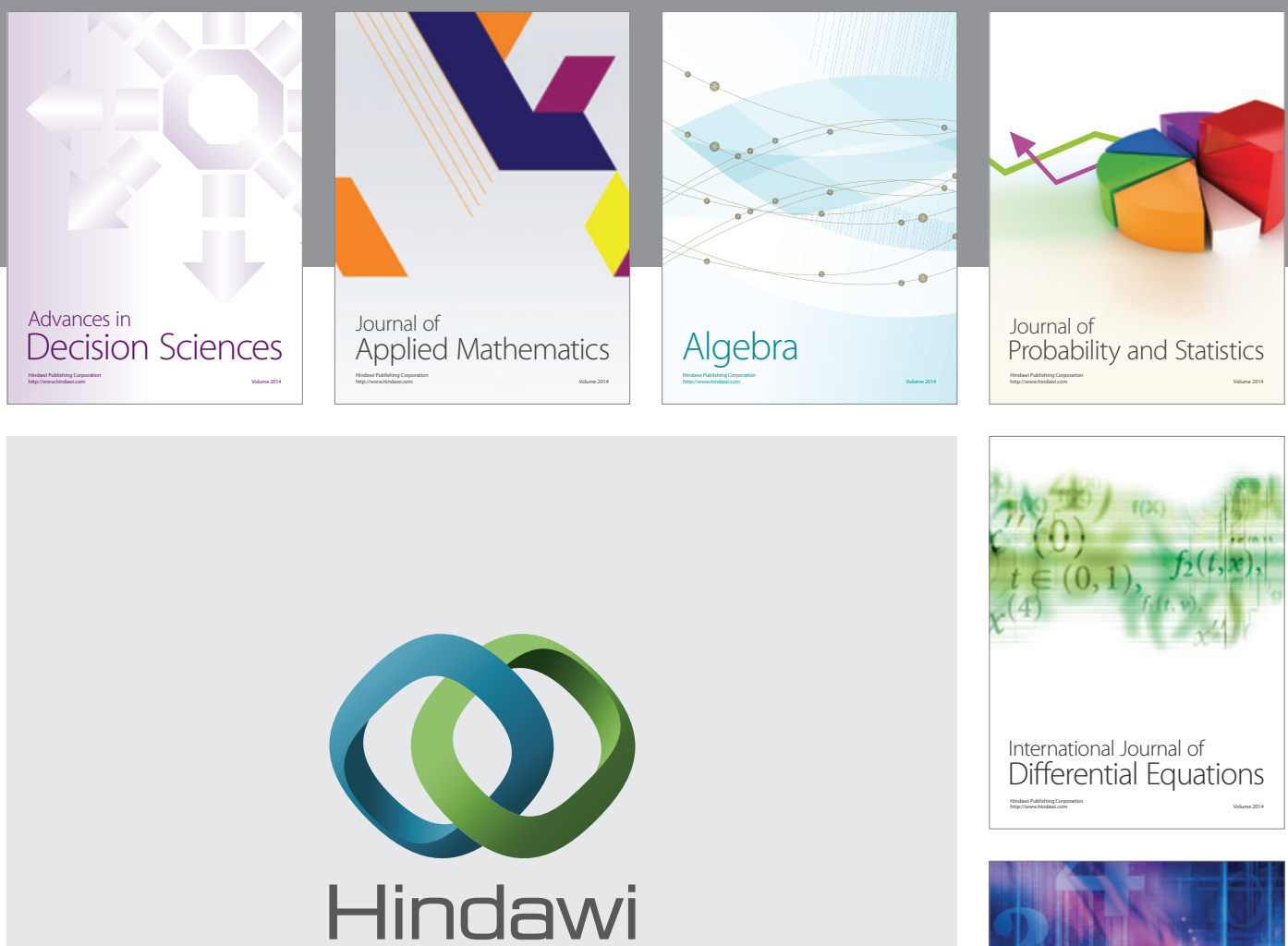

Submit your manuscripts at http://www.hindawi.com
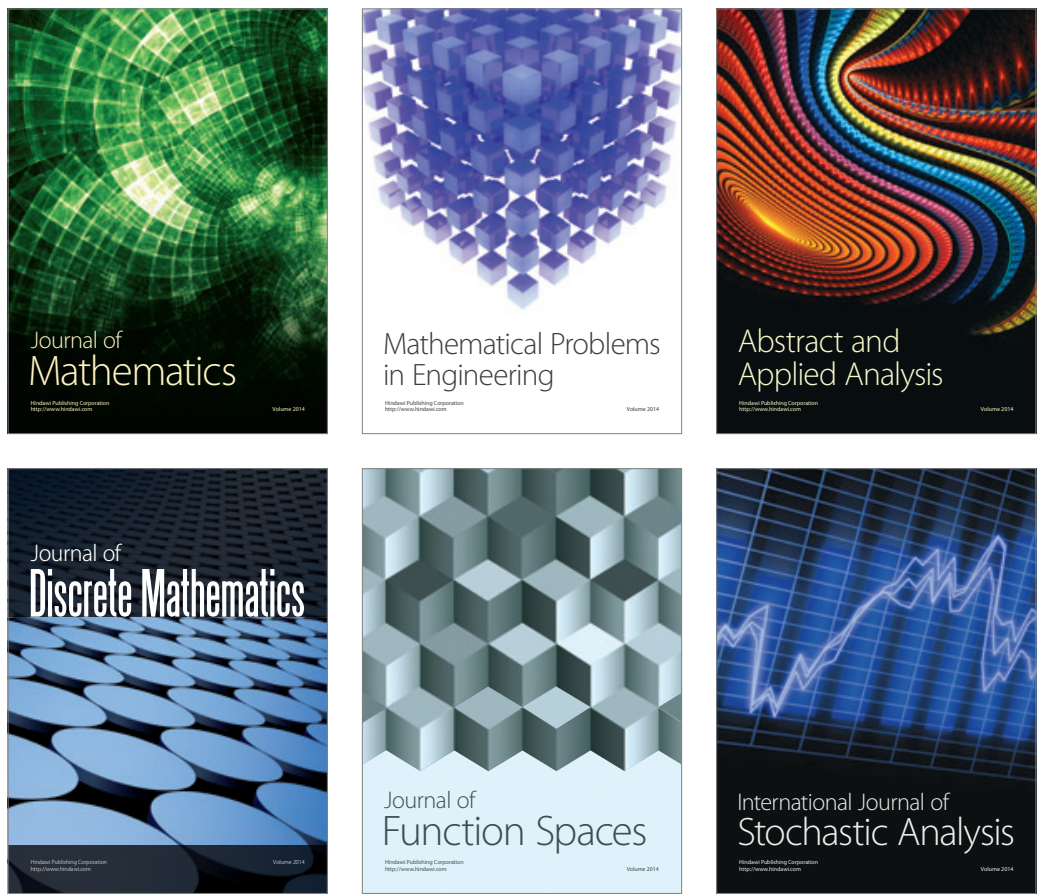

Journal of

Function Spaces

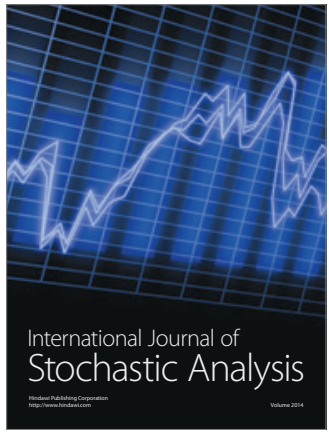

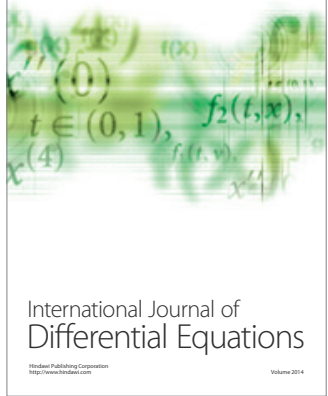
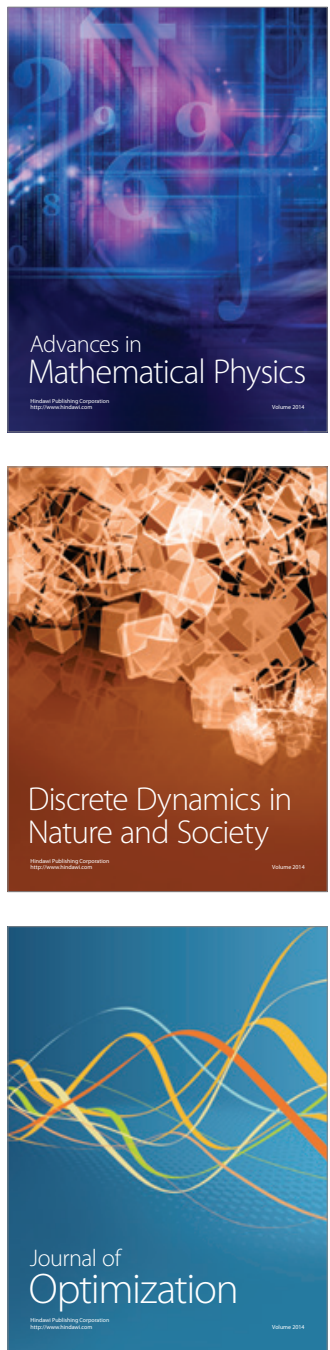